\title{
Research on rumor early warning mechanism based on kinetic energy
}

\author{
Li Wang ${ }^{1}$, Fengming Liu $^{1,}$ a , Rongrong Yang ${ }^{2}$ \\ ${ }^{1}$ School of Management Science and Engineering, Shandong Normal University, Jinan, 250014, \\ China \\ ${ }^{2}$ Department of Travel Service and Management,Technician College of Jinan, Jinan, 250014, China \\ a email: Liufm69@163.com
}

Keywords: network rumors; early warning model; measures

\begin{abstract}
Network rumors have become a severe scourge disturbing people's network life, even endangering social stability and national security. The best way to control rumor propagation is to discover earlier, so as to take actions to govern rumor before it breaks out. In this paper, from the aspects of rumor itself and rumor's audiences, taking rumor's importance and the number of spreaders as well as social environment into consideration, we propose a early warning model of network rumors based on kinetic energy. The kinetic energy is used to measure a message's attraction. When the attraction is beyond the threshold, some measures should be taken to monitor this message. If this message is judged as a rumor, the government should control the message at once and publish official information at the same time. Several experiments were conducted to verify the model's accuracy and effectiveness.
\end{abstract}

\section{Introduction}

With the development of Internet, Twitter、Wechat and QQ all have become the essential tools for instant communication. As information dissemination is no longer just relying on newspapers, radio, television and other traditional media, we have gradually entered the self-media age. Twitter 、 Wechat and other micro-propagation method have become the mainstream of information dissemination which drastically changes the communication ecology and public opinion pattern of information on the Internet. As one of the information form network rumors, spreading rapidly in the micro networks, have become a severe scourge disturbing people's network life, that's even endangered social stability and national security.

From 2005 to the first half of 2011, network rumors according to statistics have existed widely in BBS、Twitter and Blog, with the mode of transmission changing from one-dimensional propagation to multidimensional stereo propagation. As Twitter、Wechat and micro-blog are being used universally, social media has become a new platform for the propagation of rumors. The heat of keyword "rumor" has been over 30 million in Sina. Network rumors are so rampant that it has caused the attention of every state. Nations around the world develop and improve relevant laws and regulations in order to govern rumors. Germany is the first country to promulgate network written laws. From 2010, America took the lead to set up the "Social Media Capacity" project, in which "Twitter", "Facebook" and other social networking sites and their opinion leaders are under normal monitor of governments. Recently, America starts another project, called "network identification strategy", which devotes to set up a corresponding identification system in big data era and try to enhance the transparency of the network and avoid fraud, infringement and other illegal acts in virtual society. In order to strengthen the management of network service providers, Singapore, Japan and other countries establish special laws on the basis of the existing legal provisions to form industry constraints. Real-name mechanism was carried in Korean. The central network security and information leading group was set up on February 27, 2014 in China, which stand the development and management of new media to be improved to the highest top-level design. Network security has a profound impact on national security and social stability. As mentioned above this reflects the importance and necessity of rumor governance. The best way to control rumor propagation is to discover rumor earlier so as to take actions to govern rumor before 
it breaks out. In this paper, from the aspects of rumor itself and rumor's audiences, taking rumor's importance, the number of spreaders and social environment into consideration, we proposed a network rumor early warning model based on kinetic energy. In this model, kinetic energy is used to measure messages' attraction. When a message's attraction is beyond the threshold, some measures should be taken to monitor this message. If this message is judged as a rumor, the government should control the message at once and publish official information. The model is proved effectively to rumor early warning by several experiments.

Nations around the world developed early warning systems to reduce the impact of tropical cyclones, flooding, tornadoes, drought, severe hail, tsunamis and other hazards that threatened their citizens[1]. Rumors have been another hazard that threatens national security and social stability. Because of the similarity between rumor propagation and virus propagation, the majority of rumor spreading models is based on the classic epidemic models, including SI model[2], SIS model[3] and SIR model[4]. Nekovee et al. [5] introduced the forgetting mechanism into the classical SIR model and the forgetting rate was considered to be a constant. Zhao et al. [6] extended the model on the basis of Ref. [5] in which the remembering mechanism was taken into account. Their research in Ref. [7] showed that when the forgetting rate is a variable, the final size of the rumor is much larger than the forgetting rate is assumed as a constant. Though rumors and epidemics are considered as similar, there are quite lots of differences between the mathematical models for them. R.E.Dickinson et al. [8] showed that both rumor and epidemics can be subsumed under a general class of transient processes. Some researchers from different aspects study rumor dissemination. Moreno et al. [9-11] formalized a rumor spreading model using a stochastic approach on a scale-free network, which found that the rumor spreading mechanism was greatly influenced by the uniformity of the network. Ray [12] obtained and compared the size of a rumor using both deterministic and stochastic approximations in two models, one due to Daley and Kendall[13], the other due to Maki and Thompson[14]. Zanette [15,16] analyzed the dynamical behavior of rumor spreading on a small-world network. His research showed that there exists a threshold beyond which the rumor is not disseminated. George Giakkoupis et al. [17] proposed a dynamic graph model, unlike most previous works taking the topologies as static, an adversary allowed to rewire the connections between vertices before each round. Castellano et al. [18] presented a rumor spreading model by use of statistical physics. Most of the exiting rumor spreading models are on the basis of SIR epidemic model. Based on mean field theory, SIR model describes the tendency of epidemics propagation by use of differential equations, dividing people into three states, susceptible, infected and removed. However, though rumors and epidemics are popularly regarded as similar, there exits important differences between them. Three states are inadequate to accurately analyze the mechanism of rumor propagation[19]. Furthermore, influencing factors such as information importance, subjective identification of people and the trust degree of the information media should be taken into consideration to analyze the complex mechanism of rumor propagation accurately.

\section{Research on rumor spreading mechanism based on kinetic energy}

\subsection{The relationship between rumor spreading and kinetic energy}

In physics, the energy caused by an object's movement is called the object's kinetic energy. The kinetic energy formula is as follows:

$$
E_{i}(t)=\frac{1}{2} m v^{2}
$$

Where, $E_{i}(t)$ is the kinetic energy of object ${ }^{i}$ at time $t, m$ is the mass of object $i, v$ is the speed of object $i$ at time $t$.

Network rumors are actively moving among different nodes to achieve propagation. Therefore, rumors have kinetic energy. In our model, kinetic energy is used to measure rumor's attraction. The bigger the energy, the more attractive the rumor. There are many factors influencing rumors' attraction, environment(especially emergencies), government's intervention, rumor's importance 
and the number of spreaders. In previous studies, a rumor's attraction is generally determined by the final forwarding number which is so simple that it can't simulate the dynamic changes of rumor spreading and can only describe the final result of rumors' propagation. This paper can monitor the whole process of rumor spreading by calculating rumors' kinetic energy. These dynamic changes like how long a rumor spreads and when it breaks out, can be well presented in our model. The kinetic energy is defined:

$$
K E_{i}=k m v^{2}
$$

In the formula, $K E_{i}$ is the kinetic energy of node ${ }^{i}, k$ is a regulatory factor, $m$ is the importance of a rumor, $\quad v$ is the rumor's speed.

\subsection{Model}

\subsubsection{Rumor's importance $m$, representing rumor's attraction.}

The importance of a rumor's content plays an important role in rumor's attraction. It can attract more attention if the rumor meet people's psychology or it is related to their benefit. In this paper, we use keywords weight determining method to measure rumor's importance. We pick out ten pieces of great rumor cases from countries around the world, extracting keywords and assigning weights(Table 1). When a rumor appears, matching it with the keywords, calculate its importance by adding the corresponding weights.

\begin{tabular}{|c|c|c|c|c|}
\hline time & Rumor cases & Keyword 1 & Keyword 2 & Keyword 3 \\
\hline 2006 & $\begin{array}{l}\text { America, a woman was paid } 11.3 \text { million dollars } \\
\text { for being called "swindler" on the Internet, } \\
\text { which sets a new record in this kind of cases. }\end{array}$ & reputation & right & security \\
\hline 2008 & $\begin{array}{l}\text { South Korean,actress Choi Jin-Si committed } \\
\text { suicide due to rumors that Choi, as a loan shark, } \\
\text { had lent a large sum of money to Ahn. }\end{array}$ & security & public figure & reputation \\
\hline 2008 & $\begin{array}{l}\text { India, "problem vaccine" resulted in many } \\
\text { thousand children missing vaccinated. }\end{array}$ & security & medicine & children \\
\hline 2009 & $\begin{array}{l}\text { Thai, a stock trader spread rumors that the king } \\
\text { of Thai was quite ill which accounted for shares } \\
\text { collapsing. }\end{array}$ & economy & public figure & economy \\
\hline 2010 & $\begin{array}{l}\text { China, several millions people took refuge in } \\
\text { streets because of earthquake rumors. }\end{array}$ & disaster & security & economy \\
\hline 2011 & $\begin{array}{l}\text { England, occurred a large scale disturbance and } \\
\text { youths were accused for spreading rumors. }\end{array}$ & security & $\begin{array}{c}\text { social } \\
\text { atmosphere }\end{array}$ & security \\
\hline 2012 & $\begin{array}{l}\text { Mexico, a youth exaggerated the event of } \\
\text { drivers pillaging passengers into severe violent } \\
\text { crime news, which resulted in social panic. }\end{array}$ & security & terror & economy \\
\hline 2012 & doomsday & security & disaster & terror \\
\hline 2012 & $\begin{array}{l}\text { China, the rumor of toxic chicken struck KFC } \\
\text { and MacDonald }\end{array}$ & food & nation & economy \\
\hline 2014 & Malaysia Airlines flight MH370 losing contact & nation & security & terror \\
\hline
\end{tabular}

Standardizing the above keywords, according to statistics, we assigned weights for each keywords. As is shown in Table 2. 
Table 2 keywords weights

\begin{tabular}{|c|c|c|c|}
\hline keyword & weight & keyword & weight \\
\hline security & 0.33 & economy & 0.2 \\
\hline right & 0.1 & terror & 0.1 \\
\hline nation & 0.06 & disaster & 0.06 \\
\hline food & 0.03 & public figure & 0.06 \\
\hline disadvantaged group & 0.03 & social atmosphere & 0.03 \\
\hline
\end{tabular}

\subsubsection{The speed of rumor propagation.}

A rumor's speed is defined as the forwarding number of the rumor per unit time. Rumor mongers releasing rumors again and again at the initial time, when the spreaders achieve a certain scale, they stop and the spreaders usually transmit once. Assume the maximum number of rumor mongers' replication is $\mathrm{r}$. As a result, the rumor's speed can be considered as the sum of spreaders and $\mathrm{r}$ per unit time. It is defined as:

$$
v(t)=\frac{Q_{i}+r}{t}
$$

Where, $Q_{i}$ is the spreaders in the network at time t, $r$ is the maximum number of rumor mongers' replication.

\subsubsection{Rumor's energy $K E_{i}$, representing the a rumor's attraction.}

Different rumors have different attractions. The bigger the energy, the more attractive the rumor. There are many factors influencing rumors' attraction, environment(especially emergencies), government's intervention, rumor's importance and the number of spreaders. This paper takes all these factors into consideration which can monitor the whole process of rumor spreading by calculating rumors' kinetic energy effectively. How long a rumor spreads and when it breaks out, these dynamic changes can be well presented in our model. The kinetic energy is defined as follows:

$$
K E_{i}=k m v^{2}
$$

In the formula, $K E_{i}$ is the kinetic energy of node ${ }^{i}, m$ is the importance of a rumor, $v$ is the rumor's speed, $k$ is a regulatory factor which is set 1 generally. When emergencies take place, $\mathrm{k}$ is greater than 1 ; when the government intervenes, $\mathrm{k}$ is smaller than 1.

\section{Experiments}

In this section we first extract a data set from Sina involving 5000 people. Secondly, we set thresholds according to the importance of rumors. Finally, we discuss the impacts of rumor's importance and government's intervention on rumor's energy so as to explore the early warning mechanism for network rumors.

\subsection{Setting threshold $E_{0}$.}

$<$ The specification on issues of applicable law in handing libel criminal cases using information network> was issued by Chinese Supreme Court and Supreme People's Procuratorate in September 2013. The document clearly states that a libel information which is clicked and skimmed for more than 5000 times or forwarded more than 500 times that should be considered as "circumstances of aggravation" constituting crime of defamation according to Article 246 of Criminal Law. Therefore, this paper set the threshold of a rumor's energy as the message is forwarded for 500 times. 


\subsection{The early warning effects of rumor's threshold.}

The experiment explores the changing trend of rumor's energy under $\mathrm{m}=0.2, \mathrm{~m}=0.5, \mathrm{~m}=0.8$ respectively. According to formula 4, calculate the rumors' thresholds. $\mathrm{m}=0.2, \quad E_{0}=50000 ; \mathrm{m}=0.5$, $E_{0}=12500 ; \mathrm{m}=0.8, E_{0}=20000$. The result is shown in Fig.1.

The experiments show that a rumor's energy is small at the initial time no matter how important the rumor is, that is to say the attraction of a rumor is small at the beginning. When a rumor's energy breaks through the threshold, its energy improves in a straight line. At this point, the rumor erupts with the rumor's attraction greatly increased and the rumor's influence on people immensely strengthened. The more important a rumor is, the sooner it erupts and the bigger energy it has at last.

\subsection{The control effects of the government.}

As shown in the last experiment, we can see a rumor erupts when its energy breaks through the threshold. In this experiment, the government intervenes as soon as the energy breaks through the threshold aimed to explore the government's control effects. The results are shown in Fig.2.

The experiment shows that because of the timely intervention of the government, the rumor's energy rises after it breaks through the threshold, but sharply reduces to zero. It indicates that if the government discovers rumors and intervenes in the rumor's propagation as soon as possible, rumors can be well controlled.

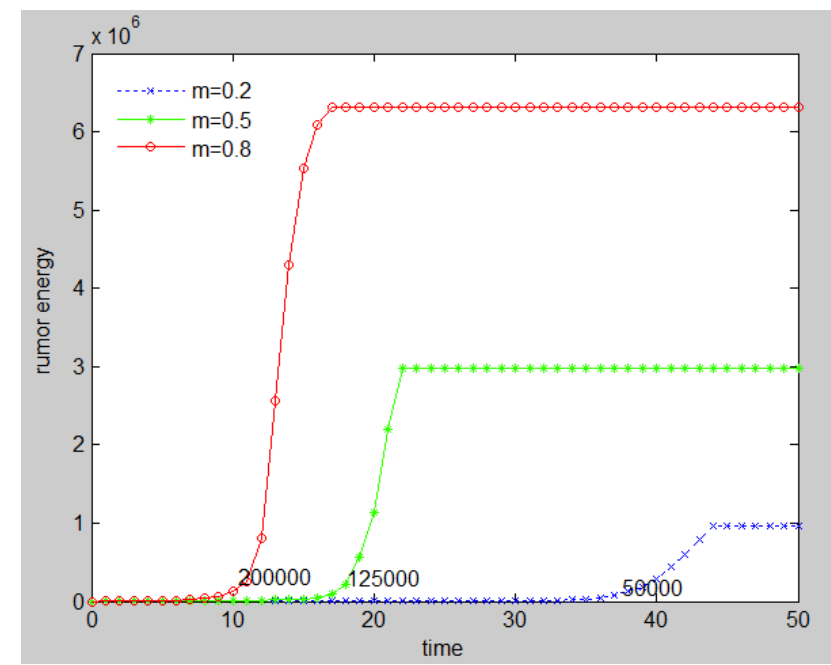

Fig.1 The early warning effects of rumors’ thresholds

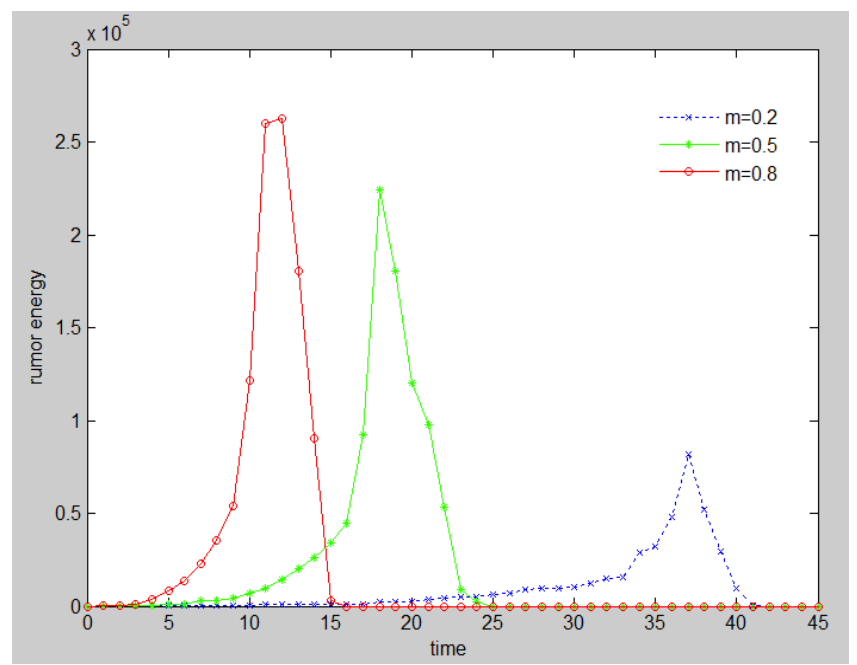

Fig.2 The control effects of the government

\section{Conclusions}

From the aspect of rumor itself, this paper proposed a rumor early warning model based on kinetic energy, in which rumor's importance, spreaders and environment are all taken into consideration. Kinetic energy of a rumor is used to measure the rumor's attraction. The paper explores the early warning mechanism for network rumors by discussing the early warning effects of rumors' thresholds and the control effects of the government. The experiments show that (1) a rumor's energy increases in a straight line as it breaks through its threshold; (2) rumors can be well controlled if the government intervenes as soon as possible. These can be effective in rumor early warning. Calculating the kinetic energy of messages in the network, if the energy is greater than its threshold, actions should be taken to monitor the message. Once confirming a rumor, corresponding departments should control the message at once and publish official information. 


\section{Acknowledgment}

This work was supported partly by the National Natural Science Foundation of China (No. 61170038, 61472231), the National Social Science Foundation of China (No. 14BTQ049), the Natural Science Foundation of Shandong Province (ZR2012FM013), and a project of International Cooperation in Training of Excellent Backbone Teachers for Advanced University in Shandong Province.

\section{References}

[1] Satoshi Kagami,Tomonobu Kitagawa,Koichi Nishiwaki,Tomomichi Sugihara,Masayuki Inaba,Hirochika Inoue. A Fast Dynamically Equilibrated Walking Trajectory Generation Method of[1] Harold Jim Keeney Jr., Steve Buan, Laura Diamond. Multi-hazard early warning system of the United States national weather service. <Institutional Partnerships in Multi-Hazard Early Warning Systems> pp.115-156 @ Springer-Verlag Berlin Heidelberg 2012.

[2] Pastor-SatorTas, R,Vespignani, A Epidemic Spreading in Scale-Free Networks [J]. Physical Review Letters 2001,86(14):3200-3203.

[3] Eguiluz V M,Klemm, K.. Epidemic Threshold in Structured Scale-Free Networks[J].Physical Review Letters,2002, 89(10):108701.

[4] Moore C, Newman, MEL Epidemics and Percolation in Small-world Network [J].Physical Review E, 2000, 61(5): 5678-5682.

[5] M. Nekovee, Y. Moreno, G. Bianconi, M. Marsili, Theory of rumour spreading in complex social networks, Physica A 374 (2007) 457 - 470.

[6] L. Zhao, J. Wang, Y. Chen, Q. Wang, J. Cheng, H. Cui, SIHR rumor spreading model in social networks, Physica A 391 (2012) 2444 - 2453.

[7] L. Zhao, W. Xie, H.O. Gao, X. Qiu, X. Wang, S. Zhang, A rumor spreading model with variable forgetting rate, Physica A 392 (2013) 6146 - 6154.

[8] R.E.Dickinson, C.E.M. Pearce, Rumors, Epidemics, and Process of Mass Action: Synthesis and Analysis. Mathematical and Computer Modeling 38(2003) 1157-1167.

[9] Y. Moreno, M. Nekovee, A. Pacheco, Dynamics of rumor spreading in complex network, Physical E 69 (2004) 066130.

[10] Y. Moreno, M. Nekovee, A. Vespignani, Efficiency and reliability of epidemic data dissemination in complex networks, Physical E 69 (2004) 055101.

[11] Y. Moreno, R. Pastor-Satorras, A. Vespignani, Epidemic outbreaks in complex heterogeneous network, Eur. Phys. J. B 26 (2002) 521 - 529.

[12] Ray Watson, On The Size Of A Rumor. Stochastic Process and their Applications 27 (1988) 141-149.

[13] Daley and D.G. Kendall, Stochastic rumors, J. Inst. Maths. Applics. 1(1965) 42-55.

[14] Maki and M. Thompson, Mathematical Models and Applications . Prentice-Hall, Englewood Cliffs, NJ, 1973.

[15] D.H. Zanette, Critical behavior of propagation on small-world networks, Phys. Rev. E 64 (2001) 050901.

[16] D.H. Zanette, Dynamics of rumor propagation on small-world networks, Phys. Rev. E 65 
(2002) 041908.

[17] George Giakkoupis, Thomas Sauerwald, Alexandre Stauffer, Randomized Rumor Spreading in Dynamic Graphs. ICALP 2014, Part II, LNCS 8573,495-507.

[18] C. Castellano, S. Fortunato, V. Loreto, Statistical physics of social dynamics, Modern Phys. 81 (2009) $591-646$

[19] Nan Zhang, Hong Huang *, Boni Su, Jinlong Zhao, Bo Zhang, Dynamic 8-state ICSAR rumor propagation model considering official rumor refutation. Physica A 415 (2014) 333 - 346 